\title{
Interview with Peter Sarnak
}

\author{
Michael Th. Rassias (University of Zürich, Switzerland)
}

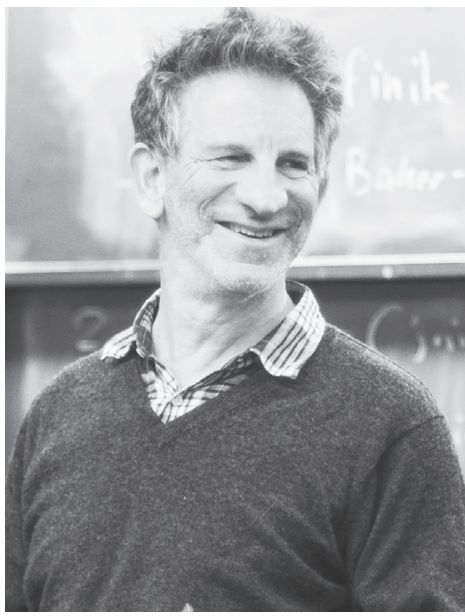

Peter Sarnak.
Peter Sarnak is the Eugene Higgins Professor of Mathematics at Princeton University, as well as a Professor at the School of Mathematics of the Institute for Advanced Study, Princeton. Born in 1953 in Johannesburg, South Africa, he studied at the University of Witwatersrand, obtaining a BSc in 1974 and a postgraduate $B S c$ (Hons) in 1975.

He obtained his PhD from Stanford University in 1980 under the direction of Paul Cohen.

Sarnak is known for his groundbreaking contributions to number theory and problems of mathematical analysis motivated by number theory. The techniques he employs in his research, as well as his interests, are surprisingly wide-ranging, from zeta functions and automorphic forms to mathematical physics and quantum computation.

Throughout his career, he has received several prestigious awards, including the Pólya Prize (1998), the Ostrowski Prize (2001), the Levi L. Conant Prize (2003), the Cole Prize (2005) and the Wolf Prize (2014), and has been awarded with honorary doctorates from several universities. He has also been elected as a member of several important academies and societies, including as a Member of the National Academy of Sciences (USA) and a Fellow of the Royal Society (UK). Peter Sarnak has also supervised more than 50 PhD students!

M.Th. Rassias: If I remember correctly, as a high school student you were very much involved with chess competitions and you did not get really interested in mathematics until your undergraduate years. Is this related to how mathematics was taught to you in your early life in South Africa or was it just a matter of taste at the time? Provided with other inputs, might you have been participating in mathematics problem-solving competitions during your high school years instead?

P. Sarnak: The mathematics that I was exposed to in high school in South Africa in the late 1960s was mostly routine and, while it came easily to me, I wasn't aware of any of the challenges that mathematics had to offer. On the other hand, chess was a challenge and one that was decided with an immediate outcome. I was drawn to it from an early age and probably hit my peak aged 16. My father was very supportive of my involvement in chess competitions until I declared my intention of going abroad af-

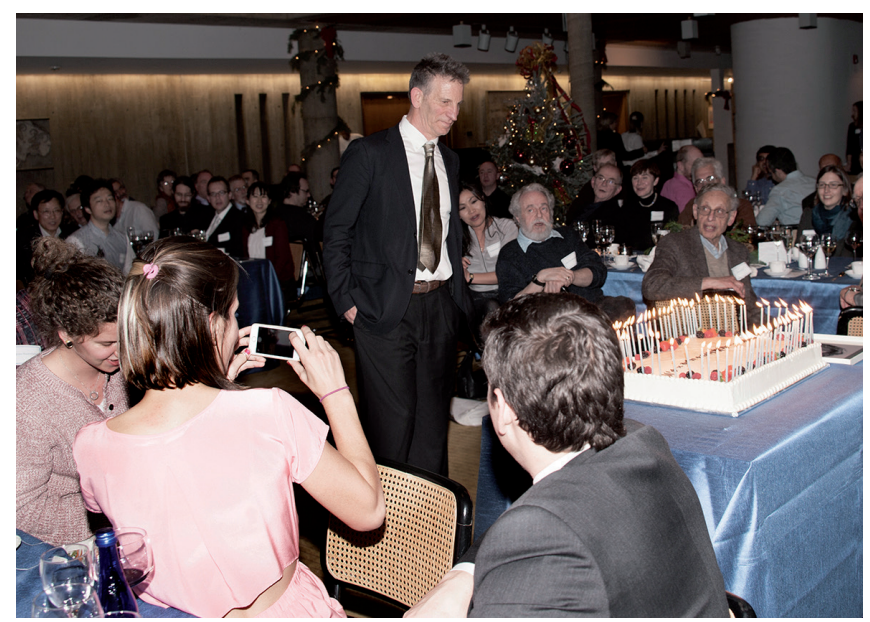

Celebration at the conference in honor of Peter Sarnak's 61st birthday at Princeton.

ter school and trying to make it as a professional chess player. He insisted that I go to university first to study and, indeed, once I got there (at the University of Witwatersrand in Johannesburg) and was introduced to real mathematics and especially abstraction, I was quickly drawn to its beauty and challenge. I carried on playing chess competitively until my early 20 s but, after that and until today, mathematics has been my passion.

Were you exposed to any other scientific disciplines before choosing mathematics? (If yes, was that beneficial?) As I mentioned, the subjects that came easily to me in high school were mathematics and science (the latter meaning basic physics and chemistry). Being a professional mathematician was not on my radar (or of those around me). So, I was planning to major in physics. However, in my first year, I had a miserable time in the laboratory and a good friend of mine Eddie Price, who was one of the top chess players in South Africa at the time and also a lecturer in the physics department of the university, told me that if it is the theoretical side of physics that I enjoy then I would be better off doing applied mathematics. So, I majored in mathematics and applied mathematics and, while it is "pure" mathematics that has driven my main interests and research, my early introduction to applied mathematics played a key role in me having broad mathematical interests and an appreciation of its applications.

Do you believe that the strategic way of thinking you cultivated as a professional chess player helped you when approaching mathematical problems and, if so, in what way?

The deductive analytic and positional reasoning, and the rich chess theory that human chess players employ (I say 


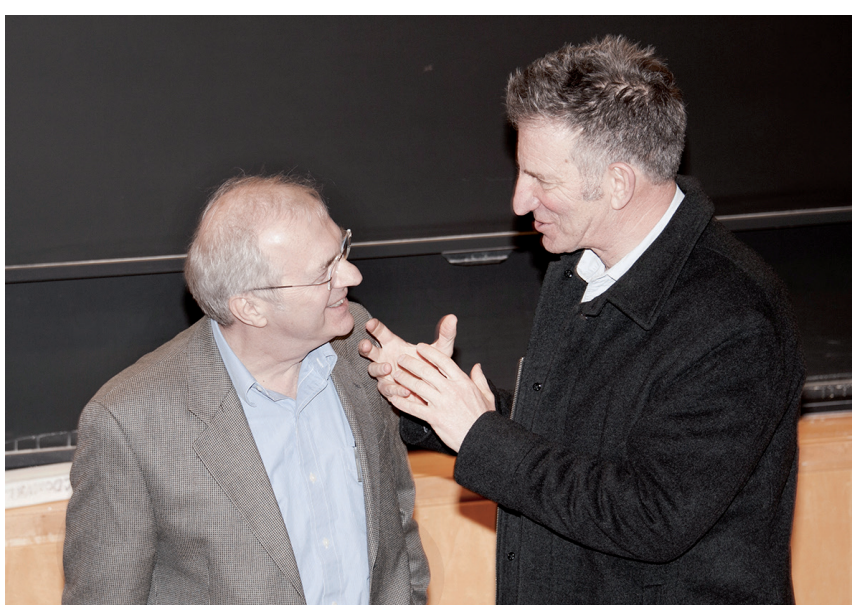

Peter Sarnak with Henryk Iwaniec.

human since, if I understand correctly, the recent computer programs such as the machine learning "alpha go", which are stronger than any program, apparently use very little "rich human chess theory"), have a lot of similarities to parts of mathematics. It is certainly very good training for the mind and for mathematics, in a similar way to mathematics competitions, but like the latter it is neither necessary nor sufficient for being a good mathematician.

Was there a specific paper, book, lecture or even theorem you came across that won you over to mathematics? What was the spark?

What won me over as a first year undergraduate studying mathematics was abstraction and specifically that conceptual thinking can make the solution of a problem and understanding of a theory completely transparent. I remember the first course in abstract linear algebra as a spark, and also a topology course that drew me to want to learn and understand much more.

One may state that mathematics has witnessed a great expansion over the last, say, 100 years, with many different areas emerging and various methods discovered, bridging seemingly different fields. You once said that: "Mathematics is really very small, not big. There aren't that many great ideas and people use the same idea over and over again in different contexts." As a mathematician who has worked in various areas of mathematics and who has used a variety of techniques in order to tackle difficult problems, do you believe that the more one matures in mathematics, the greater the unity one sees in it?

I continue to hold the opinion in the quote that you mention, though now, some years later, with the explosion of papers on the arXiv, it seems harder to hold that view. Mathematical research is looking more like other sciences, with many papers having multiple authors and even some research being done in what looks like mathematics laboratories. Some of this is natural, thanks to various tools becoming very specialised and complicated and so it is not surprising that technical projects draw contributions from people with different expertise. How- ever, when one gets down to understanding as to how an argument works, you find that the fundamental ideas and tools are much more limited than what might be apparent at first. In Hilbert's time, say, one person, like himself or his student Weyl, could have a good understanding of large portions of central mathematics. While today this might seem impossible, our mathematical universe is still small and the cornerstones from which major developments and changes are taking place at any given period are quite limited.

Is there a mathematician who influenced you the most, either through collaborations or interactions or even by studying their work?

Firstly, Paul Cohen, who was my thesis advisor, had a major influence on my mathematical taste, knowledge, insight and intuition. His view of the unity of mathematics (and that one really need not stick to a small sub-field) made a big impression on me. Together, Paul and I studied a good portion of Selberg's works, and the core of my own work is very much shaped and influenced by Selberg's ideas.

I remember you saying to us in a work group seminar at Fine Hall, Princeton, around three years ago, that you believed that the problem of factoring integers into primes must not be as hard as we think and that at some point someone will find an easy way to factor in polynomial time. Why is that?

There is no theoretical evidence that factoring is difficult and if an efficient algorithm to factor were found, no problem that is expected to be difficult (e.g. an NP complete problem) would follow. At this point in time and as far as is known in the public domain, there is no known efficient factoring algorithm, and the evidence that is offered to it being "hard" is that smart people have tried and failed. If our attitude in mathematics is that smart people have failed to solve a problem therefore it cannot be solved, we would be out of business as far as attacking the central unsolved problems.

Us mathematicians working in the trenches must have some beliefs as to what is true and what can be proved in order to proceed in our efforts, and my belief as far as factoring goes is that it can be done efficiently. In fact, one can take Shor's quantum factoring algorithm as evidence that factoring can be done efficiently with a classical computer.

Speaking about primes, you have formulated a very intriguing conjecture related to the Möbius function, which has captured the interest of many mathematicians. Would you like to describe it for the readers who do not belong to the world of number theory?

The parity of the number of prime factors of a number $\mathrm{n}$ is an elusive quantity that carries a lot of information (even the complexity of computing this parity appears to be as difficult as factoring $\mathrm{n}$ ).

If $n$ is square-free, the Möbius function $\mu(n)$ is $(-1)^{\wedge}$ (parity), while $\mu(n)$ is zero for numbers that are not square-free. As a function of $n, \mu(n)$ is apparently very 
random. For example, the partial sums of $\mu(n)$ for $n$ up to $N$ are of order roughly $\operatorname{sqrt}(N)$ if and only if the Riemann hypothesis is true. More generally, the sum of $\mu(n) . f(n)$ for $n$ up to $N$ is expected to have some cancellation for a bounded function $f(n)$ if the latter is of "low complexity"; this heuristic is known as the Möbius randomness principle and goes back at least to I.M. Vinogradov. My "Möbius disjointness conjecture" that you mention makes this heuristic precise by realising $f(n)$ as an observable sequence in a (topological) dynamical system.

If the system is deterministic (i.e. has zero entropy) then there should be cancellation. The primary tool for estimating such sums when $\mathrm{f}(\mathrm{n})$ is not a multiplicative function of $n(f(n)$ is multiplicative if $f(\mathrm{~m} n)=f(m) . f(m)$ when $m$ and $n$ are relatively prime) is Vinogradov's bilinear method. For $f(n)$ an observable in a dynamical system, Vinogradov's bi-linear sum is a Birkhoff sum for the joining of the system with itself.

This allows for dynamical ideas to be brought into the study of these sums and, as a consequence, there has been a lot of progress proving this Möbius disjointness conjecture for many deterministic systems. For logarithmically averaged versions of the conjecture, there is even more progress, thanks to works of Matomaki/Radziwill, Tao and Frantzikinakis and Host. In the latter, notions from Furstenberg's non-conventional ergodic averages and his dynamical disjointness play a central role.

It is really impressive that in your work you are able to use techniques from seemingly independent areas in order to solve a problem you are working on. In that manner, you have studied and discovered some fascinating interconnections. One example is the association of the problem of sums of squares in number theory with objects from quantum computation, such as the so-called Golden Gates. Would you like to discuss this topic?

Yes, I would be happy to elaborate on this topic. It turns out that for the construction of universal quantum gates, one is faced with the problem of providing an optimal set of topological generators for the groups $\mathrm{G}=\mathrm{SU}(2)$ and SU(4) (the first for single qubits and the second for two qubits). By optimal, we mean that the words of length $\mathrm{m}$ in the generators cover $\mathrm{G}$ (with its bi-invariant metric) optimally for all large $m$ and that there is an efficient algorithm to find the best approximation of length $\mathrm{m}$ for any $\mathrm{g}$ in $\mathrm{G}$. Standard quantum computation textbooks give specific generators for SU(2), such as "Clifford matrices plus T". It turns out that these generate an $\mathrm{S}$-arithmetic unitary group defined over a real quadratic number field and it is precisely this that makes them good generators. In recent work, Parzanchevski and I show how, for each of the (finite) symmetry groups of the Platonic solids, one can add an involution yielding an essentially optimal generating set for SU(2). Interestingly, the proof of the optimal (almost) covering property makes use of the Ramanujan conjectures established by Deligne. An heuristic algorithm developed by Ross and Selinger to navigate with the Clifford and $\mathrm{T}$ matrices can be adapted for all these "arithmetic Golden Gates", as I like to call them, and leads to efficient navigation.

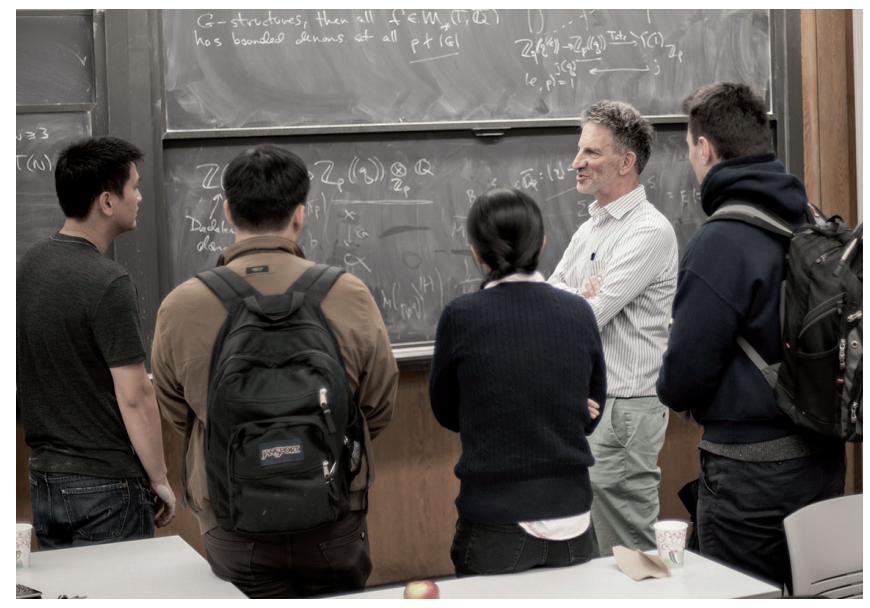

Peter Sarnak with young mathematicians.

While the mathematics of S-arithmetic unitary groups is available and provides such Golden Gates for SU(4) (Parzanchevski), efficient navigation remains open in this case.

How do you see the future of applications of number theory to other fields of science?

In Hardy's "Mathematicians Apology", he points to the theory of numbers as the epitome of "pure mathematics" and being very far from applications.

The modern digital and computer world has proven him to be quite mistaken. The applied area that is often pointed to as far as applications of number theory go is cryptography (for example, RSA and factoring). However, it runs much further than that. In fact, any fundamental problem that is discrete in nature (e.g. quantum mechanics) will, when studied down to its finest features, become one of number theory. There are many examples, such as the quantum gates above, where number theory problems that emerge are also ones that have been identified by number theorists as fundamental. I like to think that the reason for this is that in number theory (and in mathematics more generally), mathematicians are looking for the deeper truths about whole numbers and, while we are not motivated by applications, these come naturally in cases where our insights are fundamental features of the objects that we study. In any case, when things work out this way, it is particularly pleasing.

My colleague Michail Aizenman (a mathematical physicist) once commented in a lecture on random matrix theory and zeros of the zeta function that "number theory is the final frontier of science". He doesn't have to convince me of that!

Mentoring young researchers can be an important aspect of the life of a mathematician. Hilbert, for example, supervised 69 PhD students throughout his life. It is said that he enjoyed interacting with students and used to go for long walks with them to discuss mathematics. You have already supervised more than 50 PhD students and I have personally witnessed the very close relations you have with them. Would you like to talk about this aspect of your mathematical life? 
Yes, I have guided quite a number of $\mathrm{PhD}$ students over the years and I am very fortunate to have had this opportunity. For me, teaching, communicating and mentoring are an integral part of doing mathematics. Very often, I learn as much from these exchanges as do those being mentored. Over time, this reciprocal activity of guiding many $\mathrm{PhD}$ students has allowed me to learn and appreciate a much wider landscape of mathematics and it has opened doors to finding unexpected connections between disparate areas.

Directly and indirectly, the students I have mentored have played a big role in what I have managed to do. My role as a senior mentor is mostly that of being a coach: one provides encouragement and makes sure that the person being mentored is working on interesting problems and that they are aware of the basic tools that are available and what is known.

Note: The copyright of the pictures featured in this interview is held by C.J. Mozzochi, Princeton, NJ. We thank him very much for giving us the permission to publish them in this interview.

Michael Th. Rassias is on the Editorial Board of the EMS Newsletter. 